\title{
Impedimetric method for estimating the residual activity of freeze-dried Lactobacillus delbrueckii ssp. bulgaricus
}

\author{
A. Sofia Carvalho ${ }^{\mathrm{a}}$, Joana Silva ${ }^{\mathrm{a}}$, Peter $\mathrm{Ho}^{\mathrm{a}, \mathrm{b}}$, Paula Teixeira ${ }^{\mathrm{a}, *}$, \\ F. Xavier Malcata ${ }^{\mathrm{a}}$, Paul Gibbs ${ }^{\mathrm{a}}$ \\ ${ }^{a}$ Escola Superior de Biotecnologia, Universidade Católica Portuguesa, Rua Dr. António Bernardino de Almeida, P-4200-072 Porto, Portugal \\ ${ }^{\mathrm{b}}$ Departamento de Ciências de Engenharia e Tecnologia, Escola Superior de Tecnologia e Gestão, Instituto Politécnico de Viana do Castelo, \\ Avenida do Atlântico-Apartado 574, P-4901-908 Viana do Castelo, Portugal
}

Keywords: Residual activity; Lactic acid bacteria; Instrumental methods

\begin{abstract}
The residual activity of Lactobacillus delbrueckii ssp. bulgaricus cultures was analysed using $\mathrm{pH}$ and various impedimetric methods (impedance detection time (IDT), conductance and capacitance) to quantify the loss in activity following freeze-drying. The large variation recorded in IDT values for similar levels of activity suggests that IDT is not an adequate parameter for estimating the culture's fermentative activity. Comparison of the impedance signals generated revealed that capacitance yields values that are more reproducible than those of conductance, and also gives a better correlation with $\mathrm{pH}$. Statistical analysis $(p<0.05)$ indicated that there are no significant differences between the capacitance and the $\mathrm{pH}$ method when attempting to estimate residual activity.
\end{abstract}

\section{Introduction}

Lactobacillus delbrueckii ssp. bulgaricus is widely used as a lactic acid starter culture by the dairy industry. The industrial exploitation of lactic acid bacteria (LAB) as starter and/or probiotic cultures depends heavily on the concentration and preservation technologies employed, which are required to guarantee long term delivery of stable cultures in terms of viability and functional activity (Palmfeldt \& Hahn-Hägerdal, 2000). The quality of a lactic acid starter is defined by the concept of biological activity, which includes cell viability and physiological state, and is defined as ability to acidify a certain medium, and as enzymatic activities for the production of aroma and thickening (Fonseca, Béal, \& Corrieu, 2000). The activity of a particular LAB has been considered as a more suitable criterion for evaluating starter quality as opposed to its capacity to form colonies on agar medium (Fonseca, Béal, \& Corrieu, 2000); activity can be expressed as the rate of

\footnotetext{
*Corresponding author. Tel.: +351-22-5580094; fax: +351-225580088

E-mail address: paula@esb.ucp.pt (P. Teixeira).
}

(lactic-) acid production, which can in turn be used to define the effectiveness of a culture in technological processes (Lievense, Van't Riet, \& Noomen, 1990).

Activity tests for LAB are normally based on measurements of the increase in titratable acidity or the decrease in $\mathrm{pH}$ during incubation of inoculated milk for 3-5h (Okigbo, Oberg, \& Richardson, 1985). Although extensively used by the dairy industry as a standard method for the determination of fermentative activity of starter cultures, the determination of activity using $\mathrm{pH}$ is labour intensive, and the long testing times make it inappropriate for routine activity measurements. On the other hand, rapid methods, such as impedance microbiology, can provide a number of advantages over conventional methods (FirstenbergEden, 1986), because that technique provides a rapid, repeatable and accurate method for determining the shelf-life and bacteriological quality of raw materials of different food products (Pirovano, Piazza, Brambilla, \& Sozzi, 1995). Impedimetric methods have been developed to analyse a range of foods (e.g. dairy, meat and fish) and pharmaceuticals (Huist In't Veld, Hartog, \& Hofstra, 1988). These methods are based on the principle that as bacteria grow and metabolise, 
uncharged or weakly charged substrates are transformed into charged end products. The accumulation of these products leads to increases in the conductance $(G)$ of the medium and in the capacitance $(C)$ at the electrode-medium interface (Noble, Ashton, Davidson, \& Albritton, 1991).

Research in the dairy industry has primarily focused on the application of impedimetric methods for the detection of abnormal milk (Khayat \& Richardson, 1986), for the estimation of total, mesophilic and psychrotrophic counts of bacteria in raw milk (Firstenberg-Eden \& Tricarico, 1983; Nieuwenhof \& Hoolwerf, 1987), and for the selective enumeration of specific yoghurt bacteria (Pirovano et al., 1995). Impedimetric assays are regularly performed by monitoring impedance detection time (IDT). This is defined as the time required to cause a series of significant deviations from baseline impedance values, that is inversely proportional to the initial bacterial concentration (Noble et al., 1991). This parameter is a function of the concentration of microorganisms, as well as their lag phase, generation time and metabolic activity (Pirovano et al., 1995). Besides being used as a parameter for counting bacteria, conductance methods have also been used to determine lactic acid culture activity (Martos, Ruiz Holgado, Olivier, \& de Valdez, 1999). Okigbo et al. (1985) found a correlation between change in $\mathrm{pH}$ and impedance detection times, whereas Latrille, Picque, Perret, and Corrieu (1992) reported a method for real-time determination of fermentation kinetics from $\mathrm{pH}$ and conductivity data, by determining the characteristic points of the curves produced. Analysis of the changes in conductance was considered (Lanzanova, Mucchetti, \& Neviani, 1993) to be a valid method for evaluating the growth of different LAB species in milk; likewise, Svensson (1994) developed activity tests for three important bacterial groups using conductance methodologies.

The objective of this study was to investigate whether impedimetry can be used to estimate the residual activity of freeze-dried Lactobacillus delbrueckii ssp. bulgaricus. Towards this goal, various impedimetric measurements (IDT, capacitance, and conductance) were examined as appropriate parameters for estimating residual activity.

\section{Materials and methods}

\section{Bacterial strain and growth conditions}

Lactobacillus delbrueckii ssp. bulgaricus strain LB1 (hereafter termed Lb. bulgaricus) was obtained from the culture collection held at Escola Superior de Biotecnologia. The original reference cultures were maintained at $-80^{\circ} \mathrm{C}$ on glass beads. Working cultures were grown at $37^{\circ} \mathrm{C}$ for $24 \mathrm{~h}$ on de Man, Rogosa, Sharp (MRS) broth
(LAB M, Bury, UK) containing agar at the level of $15 \mathrm{~g} \mathrm{~kg}^{-1}$, as slope cultures. Slopes were stored at $4^{\circ} \mathrm{C}$ and subcultured every month.

\section{Preparation of cultures}

The MRS broth was inoculated from MRSa slopes and incubated at $37^{\circ} \mathrm{C}$ for $24 \mathrm{~h}$. This culture was then inoculated, at a level of $10 \mathrm{~g} \mathrm{~kg}^{-1}$, into a second MRS broth and incubated again at $37^{\circ} \mathrm{C}$ for $24 \mathrm{~h}$. Cells were harvested by centrifugation at $7000 \mathrm{~g}$ for $10 \mathrm{~min}$, washed twice with sterile Ringer's solution (LAB M, Bury, UK), and subsequently re-suspended in sterile reconstituted skimmed milk powder $\left(111 \mathrm{~g} \mathrm{~kg}^{-1}\right)$ (LAB M, Bury, UK). Samples of $20 \mathrm{~mL}$ were then maintained for $1 \mathrm{~h}$ at room temperature prior to freezing at $-80^{\circ} \mathrm{C}$ for $24 \mathrm{~h}$, so as to permit equilibration between the cells and drying medium, and then desiccated under vacuum at $6.7 \mathrm{~Pa}$ in a freeze-drier (Martin Christ, Osterad am Harz, Germany) for $48 \mathrm{~h}$. Four individual samples (I-IV) were then prepared.

\section{Activity measurements}

\section{Sample preparation}

For each sample, $10 \mathrm{~mL}$ of bacterial suspension prior to freeze-drying, and $10 \mathrm{~g}$ of freeze-dried culture were resuspended in 90 and $100 \mathrm{~mL}$ of sterile deionised water, respectively. Four replicates of each sample (I-IV) were used to assess the precision of each instrumental method tested.

\section{Fermenting activity using $p H$}

The decrease in $\mathrm{pH}$ after addition of the culture was measured every $15 \mathrm{~min}$ at $37^{\circ} \mathrm{C}$ in a water bath without agitation. The fermentative activity estimated using $\mathrm{pH}$ $\left(A^{\mathrm{pH}}\right)$ was defined as the maximum value for the rate of $\mathrm{pH}$ decrease per $\mathrm{mL}$ of culture before freeze-drying (BFD) $\left(\Delta \mathrm{pH} \mathrm{min}{ }^{-1} \mathrm{~mL}^{-1}\right.$ BFD), or per g of culture after freeze-drying (AFD) $\left(\Delta \mathrm{pH} \mathrm{min}{ }^{-1} \mathrm{~g}^{-1}\right.$ AFD). The maximum value for the rate of decrease of $\mathrm{pH}$ was calculated using the first derivative (at the point of inflection) of a third-order polynomial curve fitted to the data (Lievense, Van't Riet, \& Noomen, 1990). The residual activity $\left(\mathrm{RA}^{\mathrm{pH}}\right)(\%)$ was defined as the ratio of fermentative activity immediately after freeze-drying $\left(A^{\mathrm{pH}}\right)$ to the activity immediately before freeze-drying $\left(A_{0}^{\mathrm{pH}}\right)$ (Linders, Meerdink, \& Van't Riet, 1997).

\section{Fermenting activity using Bactometer}

The two components of impedance $(I)$, i.e. capacitance $(C)$ and conductance $(G)$, were measured using a Bactometer ${ }^{\circledR}$ model 64 (BioMérieux Vitek, Hazelwood Mo, USA), which was able to determine IDT automatically. A detailed description of the Bactometer ${ }^{\circledR}$ has been given elsewhere (Firstenberg-Eden \& Eden, 
1984) and only such details relevant to this study shall be discussed here. A Bactometer consists of two individual incubators, each of which contains two individual modules; each sterile disposable module, containing 16 test wells, was fitted with paired electrodes. In order to assess the repeatability of the impedance measurements, four replicates $(2 \mathrm{~mL}$ per well) of each of the four samples (both BFD and AFD cultures) were assigned to the 16 wells of each of the four modules, using a randomised complete block design (4 samples $\times 4$ replicates). The modules were incubated in the Bactometer processing unit at $37^{\circ} \mathrm{C}$ for $24 \mathrm{~h}$.

Fermentative activity estimated using conductance $\left(A^{G}\right)$ was defined as the maximum value of the rate of increase in conductance per $\mathrm{mL}$ of culture BFD $\left(\Delta G \mathrm{~min}^{-1} \mathrm{~mL}^{-1}\right.$ BFD) or per $\mathrm{g}$ of culture AFD $\left(\Delta G \min ^{-1} \mathrm{~g}^{-1}\right.$ AFD); fermentative activity estimated using capacitance $\left(A^{C}\right)$ was defined as the maximum value for the rate of increase in capacitance per $\mathrm{mL}$ of culture BFD $\left(\Delta C \mathrm{~min}^{-1} \mathrm{~mL}^{-1}\right.$ BFD) or per $\mathrm{g}$ of culture AFD $\left(\Delta C \min ^{-1} \mathrm{~g}^{-1}\right.$ AFD). The maximum velocity of increase for both capacitance and conductance was calculated as previously described in Section 2.3.2. The residual activities $(\%)$, for both conductance $\left(\mathrm{RA}^{G}\right)$ and capacitance $\left(\mathrm{RA}^{C}\right)$ after freeze-drying, were defined as the ratio of the respective activities immediately after freeze-drying ( $A^{G}$ or $A^{C}$ ) to the activities immediately before freeze-drying $\left(A_{0}^{G}\right.$ or $\left.A_{0}^{C}\right)$.

\section{Data analysis}

Statistical analysis was carried out using the statistical software R (Ihaka \& Gentleman, 1996). Pearson's product-moment correlation coefficient was used to determine the correlation between $A^{\mathrm{pH}}$ measurements and IDT. Analyses of variance (ANOVA) and Tukey's honestly significant difference (HSD) test were carried out to assess the variability (between samples) and repeatability (between replicates) of the impedimetric measurements $\left(A^{G}\right.$ and $A^{C}$ ), with a two factor ANOVA using a randomised complete block design according to notations described by Neter, Kutner, Nachtsheim, and Wasserman (1996), based on the following model:

$$
\begin{aligned}
Y_{i j k}= & \mu \ldots+\rho_{i}+\alpha_{j}+\beta_{k}+(\alpha \beta)_{j k} \\
& +(\rho \alpha)_{i j}+(\rho \beta)_{i k}+\varepsilon_{i j k},
\end{aligned}
$$

where $Y_{i j k}$ is the response variable, $\Delta G$ and $\Delta C$, for individual wells; $\mu \ldots$ the constant; $\rho_{i}$ the constant for the block (BL) effect, which were individual modules; $\alpha_{j}, \beta_{k}$ the constants for the factors, sample (S) and replicates (R), respectively; $(\alpha \beta)_{j k},(\rho \alpha)_{i j},(\rho \beta)_{i k}$ the twoway interactions; and $\varepsilon_{i j k}$ the error term.

This model assumed that there were no interactions between the block (modules) and the two-way interaction term between factors; i.e. $B L^{*} \mathrm{~S} * \mathrm{R}$ interactions were assumed to be zero. This assumption of no three-way interactions was confirmed using Tukey's test for additivity (Neter et al., 1996). Repeatability was calculated as the relative standard deviation (RSD) using the square root of the residual mean square from the ANOVA tables for $A^{G}$ and $A^{C}$, and the standard deviation for $A^{\mathrm{pH}}$. Finally, a one-way ANOVA was conducted to determine whether there were significant differences between the estimations of residual activity by the three methods $\left(\mathrm{RA}^{\mathrm{pH}}, \mathrm{RA}^{G}\right.$ and $\left.\mathrm{RA}^{C}\right)$.

\section{Results and discussion}

Lactic acid fermentation is characterised by the bacterial excretion of lactic acid into the medium. This phenomenon decreases the $\mathrm{pH}$ of the medium, and concomitantly increases its electrical conductance $(G)$ and capacitance $(C)$, as a result of the accumulation of lactate ions during fermentation (Latrille et al., 1992). The $\mathrm{pH}$ versus time curves are strictly descending, while those of conductance and capacitance changes are strictly ascending. Cultures before freeze-drying showed typical $\mathrm{pH}$, capacitance and conductance versus time curves; examples of such typical variations in $\mathrm{pH}$, conductance and capacitance with time during the fermentation of milk samples with $L b$. bulgaricus are shown in Figs. 1a and b. After freeze-drying, and due to reduction in acid production by the cultures, flat shapes were observed (data not shown). However, before and after the freeze-drying process, conductance and capacitance were inversely proportional to $\mathrm{pH}$. Considering this relationship, the method used to determine $A^{G}$ and $A^{C}$ was similar to that used to calculate $A^{\mathrm{pH}}$. Table 1 compares the repeatability of the impedimetric measurements and the $\mathrm{pH}$ for the estimation of the fermentative activity before and after-freeze-drying. The RSD is essentially the same for the three methods tested, before and after freeze-drying; however, after freeze-drying the RSD was much greater than before. These differences in RSD might have been due to the fact that freeze-dried samples were difficult to dissolve evenly. Poorer repeatability of estimates obtained between wells in a module probably resulted from solutions that bore varying degrees of solubilisation of the freeze-dried culture powder. It is not clear whether these differences result in unreliable or biased estimates of residual activity, because $\mathrm{pH}$ of freeze-dried cultures was checked for repeatability in a similar range; this point merits further consideration.

\section{$A^{p H}$ versus IDT}

Inspection of Fig. 2 indicates that IDT is inversely proportional to $A^{\mathrm{pH}}$. Although there is a significant degree of correlation $(0.77 ; p<0.0005, \mathrm{df}=14)$ between 

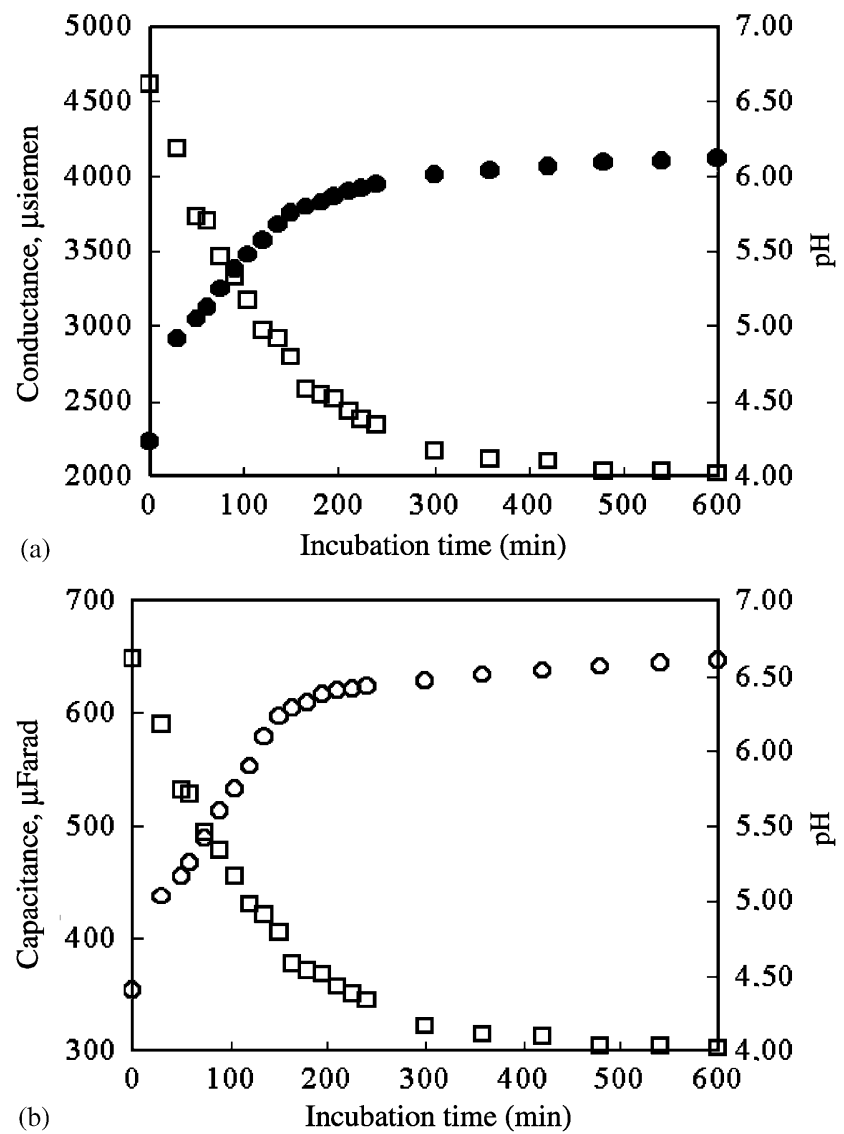

Fig. 1. Example of typical changes in $\mathrm{pH}(\square$; a, b), conductance $(\bullet$; a $)$ and capacitance $(O ;$ b) in milk inoculated with Lactobacillus bulgaricus, before freeze-drying.

Table 1

Repeatability of the calculations of fermentative activity estimated by conductance $\left(A^{G}\right)$, capacitance $\left(A^{C}\right)$ and $\mathrm{pH}\left(A^{\mathrm{pH}}\right)$

\begin{tabular}{|c|c|c|c|}
\hline & \multicolumn{3}{|c|}{$\operatorname{RSD}^{\mathrm{a}}(\%)$} \\
\hline & $A^{\mathrm{pH}}$ & $A^{G}$ & $A^{C}$ \\
\hline Before freeze-drying & 1.0 & 2.1 & 4.0 \\
\hline After freeze-drying & 32.8 & 24.4 & 26.1 \\
\hline
\end{tabular}

${ }^{\mathrm{a}}$ Relative standard deviation.

IDT and $A^{\mathrm{pH}}$ for samples after freeze-drying, the correlation between these values for samples before freeze-drying was much lower $(0.16 ; p=0.552, \mathrm{df}=14)$. Moreover, large variation in IDT values for similar values of $A^{\mathrm{pH}}$ suggests that IDT is not an adequate parameter for estimating the activity of cultures. These findings do not support the suggestion by Okigbo et al. (1985) that IDT could be used to estimate the activity of cultures based on its correlation with $\Delta \mathrm{pH}$.

The wide variability in IDT values may be due to the fact that those measurements rely heavily on the
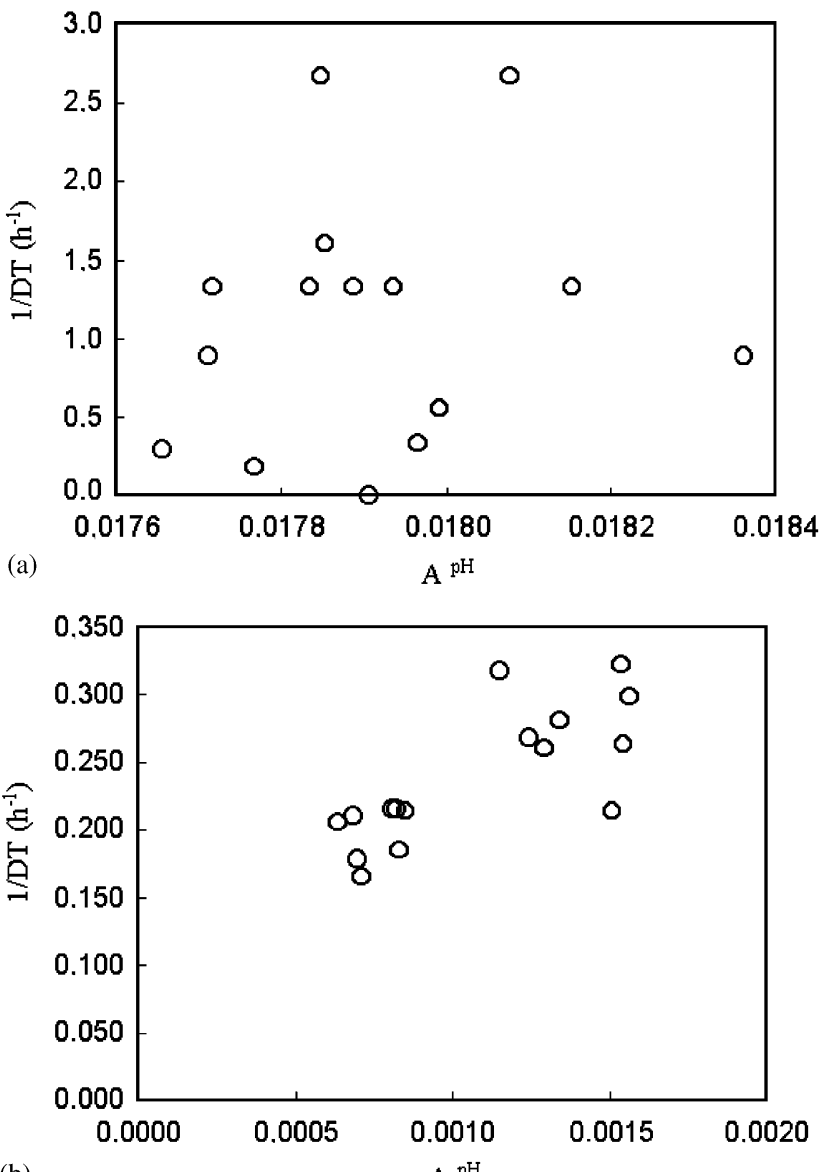

(b)

$\mathrm{A}^{\mathrm{pH}}$

Fig. 2. Comparison of the change in the rate of $\mathrm{pH}$ decrease $\left(A^{\mathrm{pH}}\right)$ in milk inoculated with Lactobacillus bulgaricus and the inverse of the impedance detection time (1/IDT), before (a) and after (b) freezedrying.

properties of the inspected impedance curves (Firstenberg-Eden \& Eden, 1984). In fact, reliable estimation of IDT can only be achieved when the impedance curves have a minimum drift and a maximum acceleration (Firstenberg-Eden, 1986). This requirement did not fully hold in our study because the cultures were very active: there was no baseline, and the acceleration phase of the impedance curves started immediately after the stabilisation time (Fig. 1). In addition, the use of skimmed milk as substrate for the activity tests may also lead to precipitation of milk proteins in the module wells during a Bactometer ${ }^{\mathbb{R}}$ run, thereby contributing to fluctuations in IDT (Lievense et al., 1990).

\section{Variability of $A^{G}$ and $A^{C}$}

Individual ANOVA were conducted for $A^{G}$ and $A^{C}$ of cultures, before and after freeze-drying, so as to compare the variability between samples (I-IV). In general, significant differences $(p<0.0001)$ were found between samples I and IV (true replicates). HSD 
Table 2

Multiple comparison of fermentative activity by Tukey's honestly significant difference (HSD) test of the variability between samples (IIV) of Lactobacillus bulgaricus before and after freeze-drying estimated by conductance $\left(A_{0}^{G}, A^{G}\right)$ and capacitance $\left(A_{0}^{C}, A^{C}\right)$

\begin{tabular}{llllll}
\hline Comparison & \multicolumn{2}{l}{ Before freeze-drying } & & \multicolumn{2}{c}{ After freeze-drying } \\
\cline { 2 - 3 } \cline { 6 - 7 } & $A_{0}^{G}$ & $A_{0}^{C}$ & & $A^{G}$ & $A^{C}$ \\
\hline I-II & $0.14^{\mathrm{a}}$ & 0.34 & & 0.02 & $0.24^{\mathrm{a}}$ \\
I-III & $0.11^{\mathrm{a}}$ & 0.03 & & $0.03^{\mathrm{a}}$ & $0.24^{\mathrm{a}}$ \\
I-IV & $0.08^{\mathrm{a}}$ & 0.01 & & $0.05^{\mathrm{a}}$ & $0.21^{\mathrm{a}}$ \\
II-III & 0.04 & 0.31 & & 0.01 & 0.00 \\
II-IV & $0.06^{\mathrm{a}}$ & 0.33 & & $0.07^{\mathrm{a}}$ & $0.45^{\mathrm{a}}$ \\
III-IV & 0.03 & 0.01 & & $0.08^{\mathrm{a}}$ & $0.45^{\mathrm{a}}$ \\
\hline
\end{tabular}

${ }^{\text {a }}$ Differences significant at $p<0.05$.

$(p<0.05)$ indicated that these differences were significant between some of the samples for only $A_{0}^{G}$ before freeze-drying, and for both $A^{G}$ and $A^{C}$ after freezedrying (Table 2). There were also significant differences between modules only for $A_{0}^{G}(p<0.0001)$ and $A_{0}^{C}$ $(p<0.001)$ before freeze-drying. A closer inspection by HSD revealed that only one module had values significantly different $(p<0.05)$ from the other three modules. This result warrants the need to pay a closer attention to variations in the estimated values from different modules. Variability among estimates between and within modules may be explained by the following factors: (i) variation in the incubation temperatures between the two separate chambers may occur, which would result in different time-temperature heating profiles between individual modules (two per chamber); (ii) a poor connection between a module and the incubator may generate unreliable signals and could lead to erroneous prediction; and (iii) individual differences between batches or between construction of modules may contribute to variability.

\section{Residual activity}

The mean residual activity after freeze-drying for each set of replicate samples (I-IV), based on the three different activity parameters examined $\left(A^{\mathrm{pH}}, A^{G}\right.$ and $\left.A^{C}\right)$ and their associated $95 \%$ confidence intervals are shown in Table 3 . The variations in residual activity were probably due to differences between the various sample replicates in the number of viable cells before and after freeze-drying, which were within the range of a single log cycle. A single factor ANOVA showed that there were significant differences between the three methods $(p<0.05)$ in estimation of residual activity. Subsequent analysis using HSD $(p<0.05)$ indicated that there was a significant difference only between the $\mathrm{pH}$ and the conductance methods when attempting to estimate residual activity (Fig. 3). A mean difference in
Table 3

Mean residual activity (\%) and 95\% confidence interval (C.I.) of Lactobacillus bulgaricus after freeze-drying, as estimated by $\mathrm{pH}$ $\left(\mathrm{RA}^{\mathrm{pH}}\right)$, conductance $\left(\mathrm{RA}^{G}\right)$ and capacitance $\left(\mathrm{RA}^{C}\right)^{\mathrm{a}}$

\begin{tabular}{|c|c|c|c|c|c|c|}
\hline \multirow[t]{2}{*}{ Sample } & \multicolumn{2}{|l|}{$\mathrm{RA}^{\mathrm{pH}}$} & \multicolumn{2}{|l|}{$\mathrm{RA}^{G}$} & \multicolumn{2}{|l|}{$\mathrm{RA}^{C}$} \\
\hline & Mean & $95 \%$ C.I. & Mean & $95 \%$ C.I. & Mean & $95 \%$ C.I. \\
\hline I & 70 & 7.3 & 44 & 8.7 & 64 & 12.7 \\
\hline II & 38 & 3.1 & 33 & 7.4 & 42 & 14.2 \\
\hline III & 47 & 1.3 & 29 & 11.3 & 43 & 3.8 \\
\hline IV & 86 & 1.1 & 68 & 7.3 & 81 & 11.9 \\
\hline
\end{tabular}

${ }^{\mathrm{a}}$ Estimation based on four replicates.

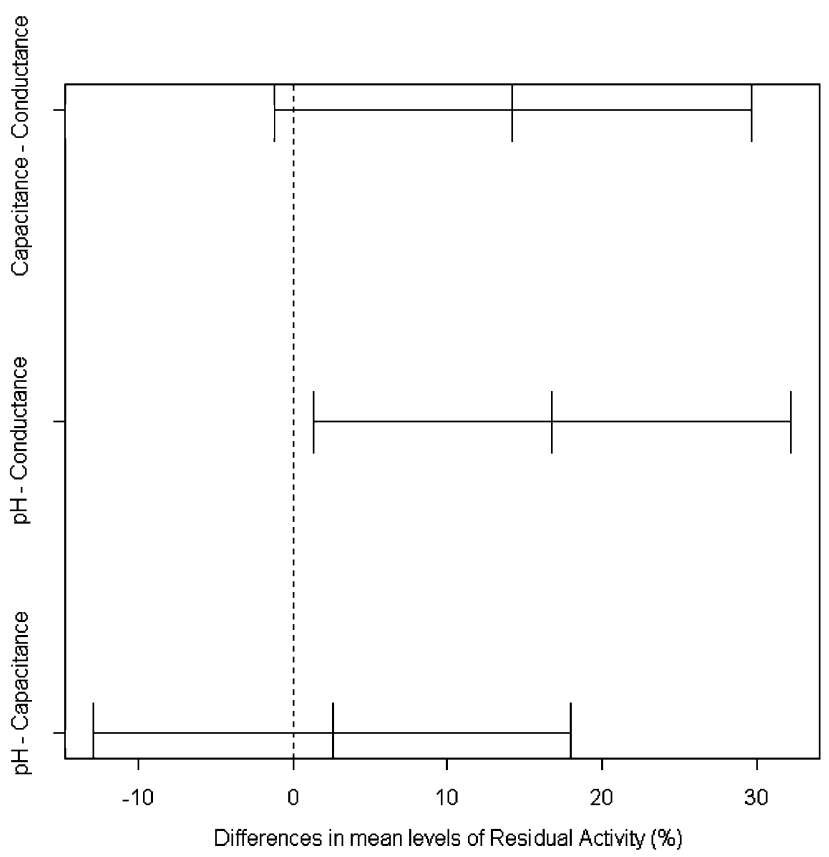

Fig. 3. Tukey's multiple comparison of the difference between mean residual activity (\%), and corresponding estimated $95 \%$ pairwise confidence interval for $\mathrm{pH}$, conductance and capacitance.

residual activity of only $2.5 \%$ was found between the $\mathrm{pH}$ and the capacitance methods.

\section{Conclusion}

The measurement of capacitance using a Bactometer ${ }^{\circledR}$ can be used as an alternative method for estimating the residual activity of freeze-dried preparations of Lb. bulgaricus. Modification of the data analysis program for the Bactometer ${ }^{\circledR}$ could be made to provide an automated means of estimating activity. This instrumental technique is fast, and allows automated collection and computerised evaluation of data from a large range of impedimetric cells. This makes it useful for routine analysis of bacterial cultures in the dairy and biotechnological industries at large, and a helpful tool in 
ensuring high quality, consistent culture performance. This study has also demonstrated the importance of using sample replicates within a module, and of using more than one module in a single incubation chamber.

\section{Acknowledgements}

This work received financial support via project PRAXIS/P/Bio/12147/1998 (FCT, Portugal), coordinated by Dr. Paula Teixeira and Dr. Paul Gibbs.

Financial support for authors A. Sofia Carvalho and Joana Silva was provided by Ph.D. fellowships, PRAXIS XXI/BD/18152/98 and PRAXIS XXI/BD/ 197131/99, respectively (FCT).

\section{References}

Firstenberg-Eden, R. (1986). Electrical impedance for determining microbial quality of foods. In M. D. Pierson, \& N. J. Stern (Eds.), Foodborne microorganisms and their toxins: Developing methodology (pp. 129-177). New York: Marcel Dekker.

Firstenberg-Eden, R., \& Eden, G. (1984). Impedance microbiology. New York: Wiley.

Firstenberg-Eden, R., \& Tricarico, M. K. (1983). Impedimetric determination of total, mesophilic and psychrotrophic counts in raw milk. Journal of Food Science, 48, 1750-1754.

Fonseca, F., Béal, C., \& Corrieu, G. (2000). Method for quantifying the loss of acidification activity of lactic acid starters during freezing and frozen storage. Journal of Dairy Research, 67, 83-90.

Huist In't Veld, J., Hartog, B., \& Hofstra, H. (1988). Changing perspectives in food microbiology: Implementation of rapid microbiological analyses in modern food processing. Food Reviews International, 4(3), 271-329.

Ihaka, R., \& Gentleman, R. (1996). R: A language for data analysis and graphics. Journal of Computational and Graphical Statistics, 5, 299-314.
Khayat, F. A., \& Richardson, G. H. (1986). Detection of abnormal milk with impedance microbiology instrumentation. Journal of Food Protection, 49(7), 519-522.

Lanzanova, M., Mucchetti, G., \& Neviani, E. (1993). Analysis of conductance changes as a growth index of lactic acid bacteria in milk. Journal of Dairy Science, 76(1), 20-28.

Latrille, E., Picque, D., Perret, B., \& Corrieu, G. (1992). Characterizing acidification kinetics by measuring $\mathrm{pH}$ and electrical conductivity in batch thermophilic lactic fermentations. Journal of Fermentation and Bioengineering, 74(1), 32-38.

Lievense, L. C., Van’t Riet, K., \& Noomen, A. (1990). Measuring and modelling the glucose-fermenting activity of Lactobacillus plantarum. Applied Microbiology and Biotechnology, 32, 669-673.

Linders, L. J. M., Meerdink, G., \& Van't Riet, K. (1997). Effect of growth parameters on the residual activity of Lactobacillus plantarum after drying. Journal of Applied Microbiology, 82, 683-688.

Martos, G. I., Ruiz Holgado, A. P., Olivier, G., \& de Valdez, G. F. (1999). Use of conductimetry to evaluate Lactobacillus delbrueckii ssp. bulgaricus subjected to freeze-drying. Milchwissenschaft, 54(3), 128-130.

Neter, J., Kutner, M. H., Nachtsheim, C. J., \& Wasserman, W. (1996). Applied linear statistical models. Chicago, USA: Irwin.

Nieuwenhof, F. F. J., \& Hoolwerf, J. D. (1987). Impedance measurement as an alternative to the plate count method for estimating the total count of bacteria in raw milk. Journal of Food Protection, 50, 665-668.

Noble, P. A., Ashton, E., Davidson, C. A., \& Albritton, W. L. (1991), Heterotrophic plate counts of surface water samples by using impedance methods. Applied and Environmental Microbiology, 57(11), 3287-3291.

Okigbo, L. M., Oberg, C. J., \& Richardson, G. H. (1985). Lactic culture activity tests using $\mathrm{pH}$ and impedance instrumentation. Journal of Dairy Science, 68(10), 2521-2526.

Palmfedt, J., \& Hahn-Hägerdal, B. (2000). Influence of culture pH on survival of Lactobacillus reuteri subjected to freeze-drying. International Journal of Food Microbiology, 55(1-3), 235-238.

Pirovano, F., Piazza, I., Brambilla, F., \& Sozzi, T. (1995). Impedimetric method for selective enumeration of specific yoghurt bacteria with milk-based culture media. Le Lait, 75, 285-293.

Svensson, U. K. (1994). Starter culture characterization by conductance methods. Journal of Dairy Science, 77(12), 3516-3523. 